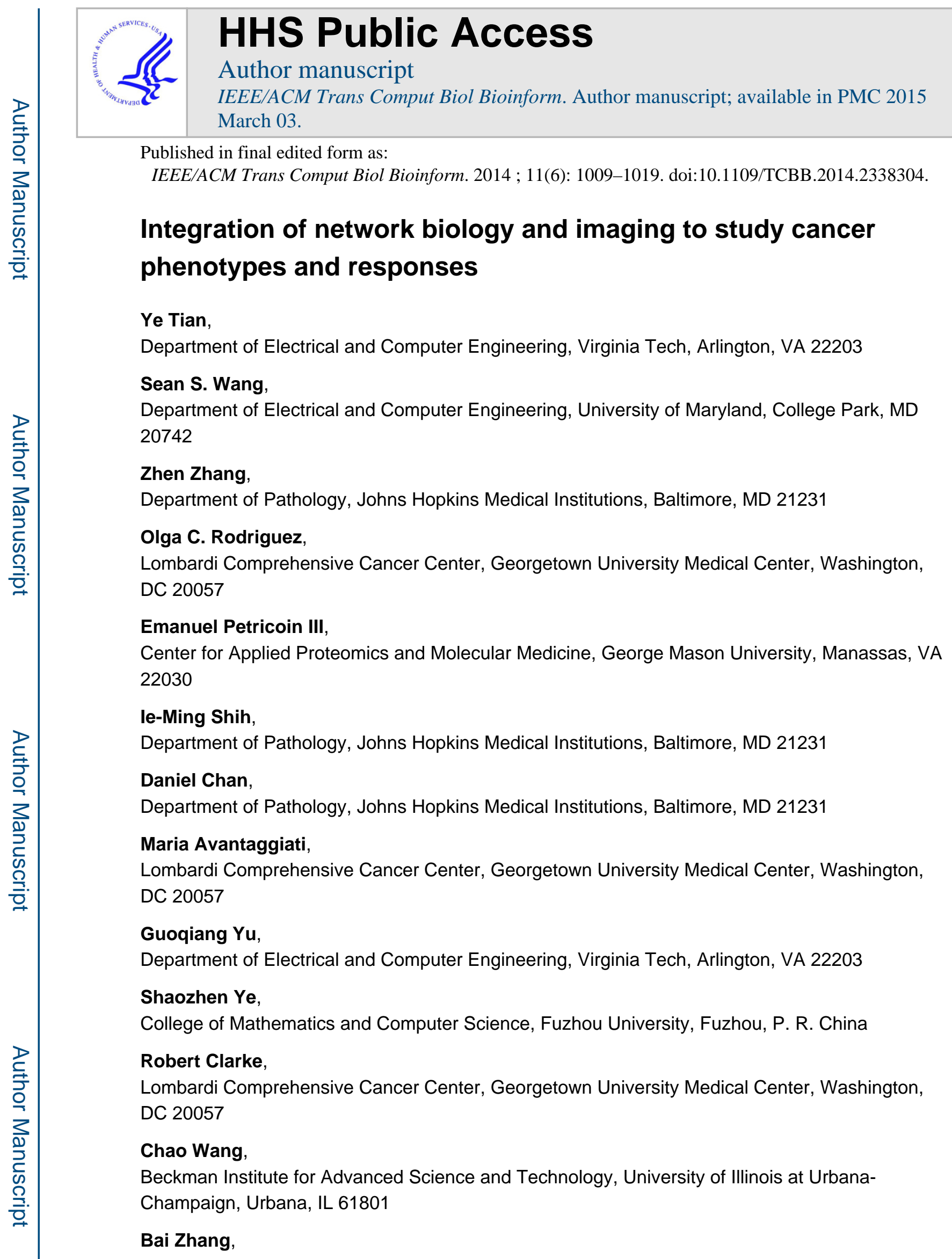

(c) 2013 IEEE. 
Department of Pathology, Johns Hopkins Medical Institutions, Baltimore, MD 21231

Yue Wang, and

Department of Electrical and Computer Engineering, Virginia Tech, Arlington, VA 22203

\section{Chris Albanese}

Lombardi Comprehensive Cancer Center, Georgetown University Medical Center, Washington, DC 20057

Ye Tian: tianye@vt.edu; Sean S. Wang: seanswang94@gmail.com; Zhen Zhang: zzhang7@jhmi.edu; Olga C. Rodriguez: rodriguo@georgetown.edu; Emanuel Petricoin: epetrico@gmu.edu; le-Ming Shih: ishih@jhmi.edu; Daniel Chan: dchan@jhmi.edu; Maria Avantaggiati: ma364@georgetown.edu; Guogiang Yu: yug@vt.edu; Shaozhen Ye: yeshzh@vip.sina.com; Robert Clarke: clarker@georgetown.edu; Chao Wang: chaowang9@yahoo.com; Bai Zhang: baizhang@jhmi.edu; Yue Wang: yuewang@vt.edu; Chris Albanese: albanese@georgetown.edu

\section{Abstract}

Ever growing "omics" data and continuously accumulated biological knowledge provide an unprecedented opportunity to identify molecular biomarkers and their interactions that are responsible for cancer phenotypes that can be accurately defined by clinical measurements such as in vivo imaging. Since signaling or regulatory networks are dynamic and context-specific, systematic efforts to characterize such structural alterations must effectively distinguish significant network rewiring from random background fluctuations. Here we introduced a novel integration of network biology and imaging to study cancer phenotypes and responses to treatments at the molecular systems level. Specifically, Differential Dependence Network (DDN) analysis was used to detect statistically significant topological rewiring in molecular networks between two phenotypic conditions, and in vivo Magnetic Resonance Imaging (MRI) was used to more accurately define phenotypic sample groups for such differential analysis. We applied DDN to analyze two distinct phenotypic groups of breast cancer and study how genomic instability affects the molecular network topologies in high-grade ovarian cancer. Further, FDA-approved arsenic trioxide (ATO) and the ND2-SmoA1 mouse model of Medulloblastoma (MB) were used to extend our analyses of combined MRI and Reverse Phase Protein Microarray (RPMA) data to assess tumor responses to ATO and to uncover the complexity of therapeutic molecular biology.

\section{Index Terms}

Network biology; MRI; differential network; cancer biology

\section{Introduction}

Cancers are often characterized by misregulation of the bio-molecular pathways that control cellular processes of DNA replication and repair, mitosis and metabolism, proliferation and apoptosis, motility and angiogenesis [1]. Viewing cancer cells as an information processing system, most cellular components exert their functions through interactions with other cellular components and are constantly making context-appropriate cell-fate decisions [2]. This interconnectivity implies that the impact of a treatment can spread along the links of the network and alter the activity of phenotypic gene products [3]. One primary interest in cancer research is how cancer cells differ from each other in their responses to treatments, where cell responses, such as signal transduction, cell-fate decisions and adaptation, are 
intrinsically context-specific $[1,4,5]$. A corollary of these views is that the changes in the interdependencies among molecular components lead to deep functional and causal relationships among apparently distinct phenotypes $[2,3,6]$. Instead of asking "which genes are differentially expressed?", further important question here is "which genes are differentially connected?" [7, 8]. Network-based approaches to cancer research have significant biological and clinical applications [3]. Compared with studies of static network topology, methods that are able to capture the dynamical changes of biological networks may reveal more insights into the critical alternations brought by cancer [6-10]. A better understanding of molecular interconnectedness on cancer phenotypes may lead to the identification of novel cancer genes and pathways, which, in turn, may offer better targets for drug development [3]. Studies on network-attacking therapies will shed new light on whether network rewiring is a general principle of cancer cells and their responses, knowledge of which would in turn be critical as molecular therapies target proteins and their networks but not genes $[2,11,12]$.

On the other hand, medical imaging applications have undergone explosive growth over the past few decades and now play multiple roles in clinical oncology [13, 14]. Medical imaging modalities provide in vivo noninvasive methods for characterizing anatomical and functional changes associated with cancer progression and responses to therapy [15, 16]. Imaging approaches have recently been used to test the efficacy of antitumor drugs by identifying new efficacy endpoints that are more easily monitored than currently used endpoints, such as invasive biopsies and survival analysis $[13,17,18]$. Furthermore, because many imaging platforms are minimally or non-invasive, longitudinal studies can be performed in a single animal, serving as an important source of phenotypic data without compromising statistical significance $[19,20]$. The truly transformative power of imaging is expected to have a major impact on individualized treatment, drug development, and response to therapy.

In this paper, we report a systems biology approach that advances our integrated magnetic resonance imaging (MRI) [21], Reverse Phase Protein Microarray (RPMA) [22] data, survival data, DNA copy number data, and Differential Dependency Network (DDN) tool $[4,5]$, collectively exploring the power of network biology (using multiplatform omics data) and in vivo imaging (deep phenomics data) to mechanistically study complex cancer phenotypes and therapeutic responses, via a well-controlled biological model system [19, $20,23]$. This work has been focused on the integration of genomics and phenomics through network biology and imaging, but not on the act of network biology or imaging in isolation $[2,17,24]$. Specifically, DDN analysis is used to detect and visualize statistically significant topological rewiring in molecular networks between two phenotypic or genotype conditions, and in vivo MRI is used to more accurately define phenotypic sample groups for such differential analysis. Unlike most existing network identification methods that focus on the network inference under a static condition, DDN emphasizes on the differential changes of network topology under different environments and their statistical significance. Finding the differential networks across multiple conditions is in essence a multitask learning problem [25], and the prior knowledge of network structure can be introduced in a Bayesian way. Knowledge incorporation in DDN is designed to equivalently tune the Laplace priors [26, 27] for the edge parameters in accordance with prior knowledge. By applying knowledge 
incorporation controlling strategy, DDN achieves maximum knowledge incorporation while staying robust to inaccurate knowledge. Further, DDN enables for the precise control of desired Type I error rate and exact p-value significance assessment for differential edges. We first demonstrate the principle and performance of DDN using realistic simulations and real gene expression data acquired from breast and ovarian cancers. To address the challenges of integrating in vivo imaging and therapeutic response data with ex vivo molecular data using a more systems-based network modeling approach to better understand the pathobiology of cancer and to better affect its cure [19,20], we provide additional new results from an ongoing preclinical drug study on Medulloblastoma (MB) demonstrating how multiscale modeling can lead to a more comprehensive understanding of cancer biology and therapy. Using Arsenic trioxide (ATO) and the ND2-SmoA1 MB model from our previous studies $[19,20]$, our current work provides the proof of principal groundwork to confirm ATO's role as an effective anti-proliferative and pro-apoptotic drug, in vivo. The outcomes and an improved understanding of ATO's mechanism of action should provide the rational basis for the design, implementation and analyses of clinical trials in children with MB.

\section{Methods}

\subsection{Overview of the Data Acquisition and Analytics Pipeline}

We developed a data acquisition and analytics pipeline to integrate multiplatform data (survival, MRI, DNA copy number, gene expression, and protein) for studying cancer phenotypes and responses to therapy. The workflow among modules and their systematic cooperation is illustrated in Fig. 1. MRI-imaging as a physical examination tool defines the phenotypic sample groups for all subsequent analysis and labels the gene expression data, RPMA protein data, survival data and tumor growth data. Survival data and longitudinal MRI-based tumor volume estimates were used to establish the efficacy of ATO treatment based on the known groups groups. Correspondingly, to corroborate with the other studies and to investigate possible mechanisms, DDN analysis uses gene expression data or RPMA protein data to detect significant rewiring of transcriptional regulatory networks between different phenotypes or drug treatments. Unlike our previous endpoint study [19, 20], and as a complete demonstration of the integrated approach, the animals on the ATO study were longitudinally interrogated by MRI for tumor growth kinetics and survival analysis, followed by DDN analysis based on RPMA protein data for mechanistic reasons of drug efficacy.

\subsection{Differential Dependence Network (DDN) Analysis}

Biological networks are context-specific and dynamic in nature [28]. Network differences among subtypes of cancer, as well as cancers undergoing treatment, provide a window into the underlying mechanisms driving the systematic changes. DDN method is used to reveal the biological networks of information processing [4, 5]. In this paper, we applied an improved DDN analytic tool that simultaneously learns conserved network structures as well as condition-specific topological changes, properly supported by the existing biological knowledge [29]. The DDN results provide great insights into the underlying biology of how 
the organisms adapt to different conditions and opening up a new era of understanding and treatment of diseases $[3,30]$.

Suppose there are $p$ nodes (genes or proteins) in the network of interest, we consider them as $p$ random variables, and denote them as $X_{1}, X_{2}, \cdots, X_{p}$. Suppose there are $N_{1}$ samples under condition 1 and $N_{2}$ samples under condition 2 . Without loss of generality, we assume $N_{1}=$ $N_{2}=N$. Under the first condition, for variable $X_{i}$, we have observations

$\mathbf{x}_{i}^{(1)}=\left[x_{1 i}^{(1)}, x_{2 i}^{(1)}, \cdots, x_{N i}^{(1)}\right]^{T}, i=1,2, \cdots, p$, while under the second condition, we have $\mathbf{x}_{i}^{(2)}=\left[x_{1 i}^{(2)}, x_{2 i}^{(2)}, \cdots, x_{N i}^{(2)}\right]^{T}, i=1,2, \cdots, p$. Further, let $\mathbf{X}^{(1)}=\left[\mathbf{x}_{1}^{(1)}, \mathbf{x}_{2}^{(1)}, \cdots, \mathbf{x}_{p}^{(1)}\right]$ be the data matrix under condition 1 and $\mathbf{X}^{(2)}=\left[\mathbf{x}_{1}^{(2)}, \mathbf{x}_{2}^{(2)}, \cdots, \mathbf{x}_{p}^{(2)}\right]$ be the data matrix under condition 2.

We use the adjacency matrix of prior knowledge network $\mathbf{W} \in \Re^{p \times p}$ to represent the prior knowledge. The elements of $\mathbf{W}$ are either 1 or 0 , with $W_{i j}=1$ indicating the existence of an edge between the $i^{\text {th }}$ gene and $j^{\text {th }}$ gene (or their gene products) in the databases such as KEGG [31], where $i, j=1,2, \cdots, p, i \neq j$. If the prior knowledge is not condition-specific, which is common in most biological databases, the prior knowledge adjacency matrix $\mathbf{W}$ will be symmetric.

Denote

$$
\mathbf{y}_{i}=\left[\begin{array}{c}
\mathbf{x}_{i}^{(1)} \\
\mathbf{x}_{i}^{(2)}
\end{array}\right], \quad \mathbf{X}=\left[\begin{array}{cc}
\mathbf{X}^{(1)} & \mathbf{0} \\
\mathbf{0} & \mathbf{X}^{(2)}
\end{array}\right]
$$

and

$$
\boldsymbol{\beta}_{i}=\left[\begin{array}{c}
\boldsymbol{\beta}_{i}^{(1)} \\
\boldsymbol{\beta}_{i}^{(2)}
\end{array}\right]=\left[\beta_{1 i}^{(1)}, \beta_{2 i}^{(1)}, \cdots, \beta_{p i}^{(1)}, \beta_{1 i}^{(2)}, \beta_{2 i}^{(2)}, \cdots, \beta_{p i}^{(2)}\right]^{\mathrm{T}}
$$

The problem of learning structural changes between two conditions with prior knowledge is formulated as a convex optimization problem. Network structures under two conditions as well as their changes are simultaneously obtained by solving the optimization problem for each node (variable) $X_{i}, i=1,2, \cdots, p$, with the objective function

$$
f\left(\boldsymbol{\beta}_{i}\right)=\frac{1}{2}\left\|\mathbf{y}_{i}-\mathbf{X} \boldsymbol{\beta}_{i}\right\|_{2}^{2}+\lambda_{1} \sum_{j=1}^{p}\left(1-W_{j i} \theta\right)\left(\left|\beta_{j i}^{(1)}\right|+\left|\beta_{j i}^{(2)}\right|\right)+\lambda_{2}\left\|\boldsymbol{\beta}_{i}^{(1)}-\boldsymbol{\beta}_{i}^{(2)}\right\|_{1} .
$$

The solution is obtained by minimizing (3), 


$$
\begin{aligned}
& \boldsymbol{\beta}_{i}=\arg \min _{\boldsymbol{\beta}_{i}} f\left(\boldsymbol{\beta}_{i}\right) \\
& =\arg \min _{\boldsymbol{\beta}_{i}^{(1)}, \boldsymbol{\beta}_{i}^{(2)}} \frac{1}{2}\left\|\mathbf{y}_{i}-\mathbf{X} \boldsymbol{\beta}_{i}\right\|_{2}^{2}+\lambda_{1} \sum_{j=1}^{p}\left(1-W_{j i} \theta\right)\left(\left|\beta_{j i}^{(1)}\right|+\left|\beta_{j i}^{(2)}\right|\right)+\lambda_{2}\left\|\boldsymbol{\beta}_{i}^{(1)}-\boldsymbol{\beta}_{i}^{(2)}\right\|_{1} \\
& \text { s.t. } \beta_{i i}^{(1)}=0, \beta_{i i}^{(2)}=0 \text {. }
\end{aligned}
$$

In (4), the structures of the graphical model under two conditions are learned jointly. The first weighted $\ell_{1}$-regularization term leads to the identification of sparse graph structure to keep the most informative connections. The second $\ell_{1}$-regularization term encourages sparse changes in the model structure and parameters between two conditions, and thereby suppresses the structural and parametric inconsistencies due to noise in the data and limited samples to highlight significant topological changes.

The problem (4) can be solved efficiently by the block coordinate descent algorithm detailed in [29]. We repeat this procedure to each node $X_{i}, i=1,2, \cdots, p$. The non-zero elements of $\boldsymbol{\beta}_{i}^{(1)}$ indicate the neighbors of the $i^{\text {th }}$ node under the first condition and the non-zero elements of $\boldsymbol{\beta}_{i}^{(2)}$ indicate the neighbors of the $i^{\text {th }}$ node under the second condition. In biological network modeling, we are particularly interested in where and how the network exhibits different topologies between two conditions. The different connections between two conditions are highlighted to indicate such changes.

The prior knowledge is explicitly incorporated into the formulation by $\mathbf{W}$ and $\theta$ in the blockwise weighted $\ell_{1}$-regularization term. A proper $\theta$ will reduce the penalty applied to $\beta_{j i}^{(c)}$ corresponding to the connection between $X_{i}$ and $X_{j}$ with $W_{i j}=1$. As a result, the connection between them will more likely be detected. A robust strategy of setting $\theta$ is used here as specified in [29] by maximizing the incorporation while controlling the degradation of performance due to inaccurate knowledge under worst case scenario (random prior) within a desired bound. Our experimental results in [29] show that such robustness is achievable. As can be expected, in the absence of definitive ground truth about the "common" and "condition-specific" biological networks, the validation of a new method for learning the networks from real data (and prior knowledge) is always problematic. Thus, we have focused our simulation studies on the impact of imperfect prior knowledge (mainly false positives), i.e., the network inference precision and recall as the false positive rate in the prior knowledge increases. Results showed that the precision and recall of inference remain robust even the prior knowledge is totaly random.

In the formulation, $\lambda_{2}$ controls the sparsity of structural and parametric changes between two conditions. DDN method uses two steps to determine $\lambda_{2}$ and assess the statistical significance of rewiring (differential edges). It is found by putting the type I error rate under null distribution in the vicinity of desired type I error rate using batches of permuted samples to get neither liberal nor conservative differential power. Suppose the network size (number of edges) is $E$ under null distribution and the expected type I error rate is $a$, i.e., about $a E$ differential edges are expected to be falsely claimed. Then, the value of $\lambda_{2}$ is determined by gradually increasing it from 0 until the empirical type I error rate matches the predesigned 
value of $a$ under the null distribution. With determined $\lambda_{2}$ we further assess the $p$-value of differential edges based on permutation test.

DDN learning uses a Block Coordinate Descent (BCD) algorithm in which we have developed a closed-form solution for sub-problems [27]. The fast solution enables scalability to both large network size and sample size. Further more, closed-form BCD algorithm can be easily paralleled and distributed, and the DDN software package also provides the users with a parallel computing option utilizing ubiquitous multi-core machines. We experimentally compared DDN with a universal convex problem solver CVX $[32,33]$, where DDN reaches a stable solution about 600 times faster than the CVX solution. According to our records, on a computer with $2.3 \mathrm{GHz}$ CPU frequency, 100 runs of DDN for inferring a 100-node network (with 150 samples) used about 6 seconds per run, inaverage, in contrast to 3,764 seconds per run by CVX. We also tested DDN using 1000 nodes and 1000 samples. When accurate $p$-value on each of the differential edges is required, a sufficient number of permutations will be used to estimate the null distribution. The computational-intensive nature of the permutation sampling will be the only limiting factor that tightly depends on the sample size [29]. Nevertheless, since DDN is aimed to detect significant rewiring between different phenotypic conditions where within-group samples must be highly "homogeneous", our experience indicates that sample size is generally not a problem in real data analysis.

\subsection{Chromosome Instability Index (CIN) Analysis}

Somatic copy number alterations are common genetic events in the development and progression of various human cancers, and significantly contribute to tumorigenesis [34, 35]. Recent advances in oligonucleotide-based single nucleotide polymorphism arrays have made it possible to detect regional amplifications and deletions with high resolution on a genome-wide scale [36]. To quantify the relative impact levels of somatic DNA copy number alterations, we used a previously described method to calculate the genome-wide chromosomal instability index (CIN) [37]. The CIN Index is defined based on CNA results as the number of segments weighted by amplitude of alteration to reflect how intense the structural variation a chromosome has gone through. For chromosome $i$, denote the largest amplitude of all samples as $A^{(i)}$ and the smallest amplitude of all samples as $D^{(i)}$. In sample $i$ there are $N_{j}^{(i)}$ segments and $n_{j, k}^{(i)}$ probes in the $k$-th segment with amplitude $m_{j, k}^{(i)}, k=1,2, \cdots, N_{j}^{(i)}$. The cutoffs of amplification and deletion are $\tau_{1}$ and $\tau_{2}$ respectively. Then the CIN of chromosome $i$ for sample $j$ is defined as:

$$
C I N_{j}^{(i)}=\frac{\sum_{m_{j, k}^{(i)}>\tau_{1}}\left(m_{j, k}^{(i)}-\tau_{1}\right) n_{j, k}^{(i)}+\sum_{m_{j, k}^{(i)}<\tau_{2}}\left(\tau_{1}+\frac{\left(\tau_{2}-m_{j, k}^{(i)}\right)\left(A^{(i)}-\tau_{1}\right)}{\tau_{2}-D^{(i)}}\right) n_{j, k}^{(i)}}{\sum_{k=1}^{k=N_{j}^{(i)}} n_{j, k}^{(i)}},
$$

where $\tau_{1}=2.5$ and $\tau_{2}=1.5$ are parameters we use here.

We use a vector to represent the chromosome instability of a sample [37]. 


$$
\mathbf{C I N}_{j}=\left(C I N_{j}^{1}, C I N_{j}^{2}, \cdots, C I N_{j}^{22}\right)^{T} .
$$

\subsection{Survival Analysis}

Survival information is used either as a criteria to define groups or to validate the effect of drug treatment. In the group division, samples with vastly different survival time were classified into two groups for further comparative analysis. In drug treatment study the survival of treated and untreated samples were compared. Once small tumors were detected by MRI, the mice were randomly divided into "ATO" and "MB" groups, and were treated with either ATO or sterile saline (the vehicle for ATO), respectively, as previously described in an end-point study of ATO $[19,20]$. The new data were collated as records of the date of birth, treatment, and death date, and multi-step and criteria quality control was performed. The samples size observed at endpoint and used in subsequent analysis are $n_{\mathrm{ATO}}=45$ and $n_{\mathrm{MB}}=41$. To assess the efficacy of ATO treatment, we performed Kaplan-Meier survival analysis, generated the survivorship curves of the mice, and calculated the P-values and hazard ratios at $95 \%$ confidence intervals (Fig. 2), consistent with our previous findings.

\subsection{MRI Volume Based Tumor Growth Rate Estimation}

Using MRI, we have previously reported that ATO was effective at blocking tumor progression in the ND2-SmoA1 mouse model [23] and that ATO affected tumor metabolic activity using magnetic resonance spectroscopy [19]. The imaging datasets were acquired using the Georgetown-Lombardi Preclinical Imaging Research Laboratory's 7.0 Tesla Bruker MRI with a $35 \mathrm{~mm}$ birdcage brain imaging coil. The mouse was placed in our proprietary stereotaxic holder with temperature and respiration control (Fig. 3). $T_{2}$-weighted RARE sequence is used with image matrix $=256 \times 256$; TR $=4660 \mathrm{~ms}$; TE $=36 \mathrm{~ms}$; RARE factor $=8$; averages $=4 ; \mathrm{FOV}=3.5 \mathrm{~cm}$, slice thickness $/$ interslice $=0.5 \mathrm{~mm}$ essentially as previously described $[19,20]$.

The MB tumors appeared as bright homogeneous regions with irregular outer edges due to tumor-induced vasculature and changes in the normal surrounding cerebellum (Fig. 3). The major limitations associated with existing manual segmentation methods are that the software drawing tools are often awkward and inconvenient and that the results can be inaccurate, subjective, and prone to human error. We adopted a semi-automatic lesssubjective approach, a novel combination of nearest-neighbor region growing and Canny edge detector [21,38], to accurately define tumor boundaries and estimate tumor volume in MRI datasets. The volume data from tumor-bearing mice, with $\left(n_{\mathrm{ATO}}=5\right)$ or without $\left(n_{\mathrm{MB}}=6\right)$ ATO treatment were collected via longitudinal MRI scans taken at 2 4 time snapshots separated by days, resulting $60-120$ scans. Due to limited time points and observed linearity in most samples, we fit a linear regression model to the time-course of tumor growth data to estimate the overall tumor growth rates and performed two-sample unequal-variance significance $t / U$-tests to assess the efficacy of ATO treatment. 


\subsection{Protein Microarrays and Differential Analyses}

Using tumor samples from these mouse models, we have previously performed RPMA [22] to define the protein expression profiles in treated versus control MB tissues [19]. Our original proteomics analyses were initially focused on a few proteins involved in primarily receptor signaling and cell cycle regulation [19]. Further analyses were performed to encompass many proteins covering abroad spectrum of the proliferative, apoptotic and intracellular signaling pathways. A total of $154 \mathrm{QC}$-approved cell cycle relevant proteins were assayed using RPMA. Both two-sample unequal-variance $t$-tests and permutationbased significant test were performed to identify significantly differentially-expressed proteins between the ATO and MB control groups.

\section{Results}

\subsection{Simulation Study Demonstrates the Performance of DDN}

As an illustrative and validation example, we first demonstrated the performance of DDN in discovering both common and unique edges between conditions using a realistically simulated data set. We used SynTReN [39], a gene expression data simulator, to simulate the expression value of 20 genes under two conditions. The ground-truth network is shown in Fig. 4(b), with red and green edges indicate condition-specific connections and black edges indicate edges shared by both conditions. The DDN learning results are shown in Fig. 4(a). Out of 10 differential edges, 7 are correctly identified by DDN and no false positive differential edges are committed. In addition, 5 shared edges are also identified. This example represents the typical usage of DDN and demonstrates the effectiveness in discovering biological network structural changes.

\subsection{DDN Finds Novel Rewiring in Study of Breast Cancer Recurrence After Treatment}

We first used DDN to find the network changes occuring in breast cancer recurrence after treatment using a public patient data [40] which are harvested on Affymetrix U133A platform. The samples were divided by phenotype feature into two groups according to recurrence status: Condition 1 (recurrent) is represented by 30 samples, in which cancer recurrence was found within 3 years after surgery. Condition 2 (non recurrent) has 37 samples, which still are recurrence free by the time data were reported and have already lived at least 9 years after surgery.

We focused on the Cell Cycle pathway retrieved from KEGG pathway database [31] as prior knowledge for the study. In the network result representation, node color indicates gene expression fold change of condition $2 /$ condition 1 . White means no change; redder means more up-regulated in condition 2 and down-regulated in condition 1; greener means more down-regulated in condition 2 and up-regulated in condition 1.

There are two types of edges, straight lines and zigzag lines, each of which has three attributes: color, arrow shape and thickness. For straight lines, red means the edge only exists in condition 1, and there is no edge in condition 2. Green means the opposite that edge only exists in condition 2 and there is no edge in condition 1. Edges with arrows indicate positive dependency (promotion) between genes. Edges with $\mathrm{T}$ arrow indicate negative 
dependency (inhibition) between genes. For zigzag lines, red lines mean positive dependency in condition 1 but negative dependency in condition 2. Green lines mean negative dependency in condition 1 but positive dependency in condition 2. For all lines thickness indicates the significance level: All edges are detected at the 0.05 significance level, but with different p-values. The thicker the edges are the smaller the p-value, and the more significant.

The differential network results of the Cell Cycle pathway are shown in Fig. 5, and identifies a differential rewiring hub with SFN (14-3-3 $\sigma$ /YWHAS), a unique member of the 14-3-3 family. SFN is more often tumor suppressive rather than pro-growth/pro-proliferative. In breast cancer, its promoter becomes hypermethylated (leading to silencing) early in during cancer progression. The inverse expression pattern for SFN and YWHAZ in these tumors, and the previously undocumented negative relationship between these two 14-3-3 family members are interesting phenomena. One could hypothesize that there is a shift in dominance between Early and Never recurrences. The $\delta$ form of YWHAZ (14-3-3 $\zeta / \delta)$ is brain-specific and $\zeta$ form binds IRS1. Increased expression of YWHAZ is already shown to be associated with poor Tamoxifen response [41, 42].

\subsection{Genomic Instability Affects Ovarian Cancer Transcriptional Network Topology}

It is believed that genetic aberrations play a role in tumorigenesis in part through further by increasing the rate of chromosome mutations, including deletion and amplification of genes involved in critical cellular processes. Chromosomal instability is a defining characteristic of most human cancers [43]. However, the role of CIN in tumorigenesis remains poorly understood and few clues exist regarding the interaction between CIN and the transcriptional networks that lead to phenotype differences [44].

We applied DDN to analyze TCGA ovarian cancer data set to evaluate how the different overall patterns of breaking points in chromosomes affect the network structure. We used the calculated CIN Index to quantify the genomic instability degrees of tumor samples. Specifically, based on the values of CIN Index, we divided the samples into groups that were genetically most unstable and most stable and compared their transcriptional network differences. We used TCGA level 3 copy number data to calculate the CIN Index for 556 samples. Each of the 22 chromosomes were assigned a CIN Index to reflect its stability in terms of number of break points and amplitude of amplifications or deletions.

We selected 25 samples with lowest CIN indices as stable group and 25 samples with highest CIN indices as the unstable group from TP53 mutated samples. Our method was applied to learn the differential network structure between stable and unstable groups in Cell Cycle and Apoptosis pathways were retrieved from KEGG pathway database and the results are shown in Fig. 6. Red edges represent the unstable group and green edges represent the stable group. The network structure in the stable group was more defined as the green edges are connected in longer paths and form complex networks. While red edges in unstable groups appear shorter and more sporadic. 


\subsection{Integrated Study on MB Responses to ATO Treatment}

Medulloblastoma is the most common and highly malignant childhood brain tumor that has a tendency to spread throughout the brain and central nervous system. Current therapies leave a majority of survivors with serious auditory and neuro-cognitive deficits, underscoring the need for the development of more effective treatments for MB. The origin of MB is complex and is often characterized by alterations in survival and cell cycle regulatory pathways and genes. For example, Sonic Hedgehog pathway is normally inactive in the mature cerebellar neuron, while its activation by the Smoothened (Smo) signal leads to unchecked tumor cell proliferation [45].

ATO is an FDA approved, second line therapeutic used for treating certain cancers and we have shown it can effectively treat MB, preclinically [23] via targeting hypothesized MB signaling mechanisms [46]. There is a significant information gap in the literature on the efficacy and therapeutic molecular biology of MB treated by ATO. Furthermore, the previous in vivo studies using the ND2-SmoA1 MB model was primarily an endpoint study, and the information on kinetics of tumor growth was missing, accurate tumor growth analyses using longitudinal imaging was lacking, and a multiplatform systems biology approach was not rigorously applied.

We acquired the new survival data, MRI volumetric scans, and protein expressions from the ND1-SmoA1 MB mice, in vivo. This combination of precise survival-imaging based phenotyping with multiplatform molecular genotyping creates an opportunity to mechanistically elucidate the efficacy and mechanism of action by ATO on MB.

Using QC-approved survival data, we performed Kaplan-Meier survival analysis. The curves in Fig. 2 give percent survival on the $y$-axis and time (in days) on the $x$-axis. Logrank test comparing the survival curves resulted in a $P$-value of $0.0499(<0.05)$, and hazard ratio of 0.6719 with $95 \%$ confidence interval. Consistent with our previous study, ATO increased overall survival with a larger mean of 181 days versus a mean of 161 days in control treated animals.

MRI based tumor volume estimates are summarized in Table 1. The ATO group had a mean growth rate of $0.0116 \mathrm{~mm} /$ day with a standard deviation of $0.0047 \mathrm{~mm} /$ day; the $\mathrm{MB}$ control group had a mean growth rate of $0.0361 \mathrm{~mm} /$ day with a standard deviation of $0.0472 \mathrm{~mm} /$ day, The tumor growth curves are shown in Fig. 7. While our tumor growth results do not reach statistical significance ( $P$-value of 0.1299 by two-sample unequal-variance t-test), the combination of the survival and MRI data strongly indicated, for the first time, that ATO reduced actual tumor growth rates, resulting in an increased lifespan in ATO-MB mice. Our results also support the utility of an imaging-based strategy for assessing and monitoring tumor response to therapy, potentially valuable in clinical trials of novel therapies using human subjects where imaging signatures as the endpoint provide a non-invasive and continuous monitoring capability [18].

We had previously reported that ErbB2, a protein involved in growth of cells, was downregulated with ATO treatment [19]. Looking more extensively at the top 20 prioritized proteins (Fig. 8), we examined their up/down-regulation between ATO-treated mice and MB 
mice without treatment. We subsequently searched relevant literature to infer/confirm the roles of ATO in regulating cell cycle machinery. Our results indicated that ATO downregulated important anti-apoptotic (or pro-proliferation oncogene) protein expression. Our current analyses now indicate that ATO up-regulated/induced many proapoptosis genes, including Bad, Stat3, Smac/Diablo, $\beta$-catenin, Ask1, and cleaved Casp3. Since for all these proteins, $\mathrm{P}<0.05$, our hypothesis that the apoptotic mechanisms are triggered or proproliferation mechanism are suppressed by ATO and is statistically significant at $\alpha=0.05$.

In response to ATO treatment, different regulatory components and mechanisms are activated and the topology of the cell cycle signaling network changes accordingly [47]. Using the RPMA expression data for 154 cell cycle related proteins, we applied DDN, to detect the topological changes in protein-protein interaction (PPI) networks between MB and ATO. In a biological system or process, many genes work together in cohesion. In addition to ATO's effects directly on relevant proteins, the ATO-induced rewiring of cell cycle signaling pathway network represents another form of molecular therapeutic mechanisms. Our DDN analysis, for the first time, detected many novel topological changes in biological network between the ATO and MB mice (Fig. 9). Cyclin D1 was previously identified being targeted by ATO [19], while cPLA2 provides intracellular arachidonic acid to supply both cyclooxygenase and lipoxygenase pathways in cell growth and survival. Our DDN analysis result indicated that the connection between Cyclin D1 and cPLA2 (marked by arrow in Fig. 9) existed in MB has been destroyed by ATO treatment. MEK and EGFR are the two cascaded components of MAPK/ERK pathway in regulating transcription and translation. Our result shows that the critical link between MEK and EGFR (marked by arrow in Fig. 9) has been eliminated by ATO.

We further manually identified proteins closely involved in MB signaling and learned the DDN on this focused subset by comparing the networks of normal samples, tumor samples and tumor samples after ATO treatment, the result of which is shown in Fig. 10. Edges with different colors have unique meanings: blue - fully recovered by treatment (edges destroyed by tumor are regained); green - weakly created connection after treatment; red - not recovered; black - fully broken down tumor connection; gray - partially broken down tumor connection. Our results may provide new insights into how ATO suppress cancer cell growth at systems level. We had previously reported that ATO restored the link between IGFR1 and ErbB-2 [19]. In this more comprehensive study, DDN identified a novel ATO driven link between induced p38MAPK activity and the spindle checkpoint protein BUB3. In addition, since the link between p38MAPK and HIF1 remain partially active, further insights into the mechanistic insights into ATO anti-tumor activity are illuminated. Importantly, cPLA2 provides intracellular arachidonic acid to supply both cyclooxygenase and lipoxygenase pathways in cell growth and survival. Our DDN analysis result indicated that the levels cPLA2 activity that existed in MB have been significantly affected by ATO treatment, possibly also helping to repress HIF1 activity in the tumors. 


\section{Conclusions}

We present a framework to integrate comprehensive analyses including DDN, MRI, tumor growth modeling, survival analysis and RPMA analysis. Outputs of different analysis modules are interconnected to consolidate the overall conclusions.

DDN analysis was applied to public gene expression data of breast cancer and ovarian cancer samples, and to protein expression data of MB samples to study their network signatures and responses to drug treatment. In network comparison of breast cancer recurrent patients and non-recurrent patients, DDN identified interesting alterations in gene interactions that may play critical role in breast cancer recurrence. Further, DDN was used to reveal how genetic variation affects network structural changes in high-grade ovarian cancer data.

As a more challenging task of integrating multiple data sources into a systematical study, we presented our integrative approach in the MB treatment experiment involving sources of information with multiple scales. Using the ND2-SmoA1 MB model and novel combinations of in vivo imaging, molecular profiling, and computational biology, we confirmed our previous findings that that ATO treatment increased overall survival and went on to establish that ATO reduced actual tumor growth rates using longitudinal MRI-based tumor volume estimates in vivo. DDN analysis confirmed that treatment responses to ATO involved changes in critical protein-pathway interactions in the cancer cells proliferative machinery and revealed a novel topological rewiring of biological network by ATO, providing new insights into how ATO suppresses cancer cell growth, warranting further extensive therapeutic and mechanistic studies [3].

\section{Acknowledgments}

The MRI was performed in the Georgetown Preclinical Imaging Research Laboratory. This work was supported in part by the National Institutes of Health under Grants CA129003, CA149147, HL111362, CA164384, NS029525, and CA160036 from Clinical Proteomic Tumor Analysis Consortium (CPTAC).

\section{References}

1. Tyson JJ, Baumann WT, Chen C, Verdugo A, Tavassoly I, Wang Y, Weiner LM, Clarke R. Dynamic modelling of oestrogen signalling and cell fate in breast cancer cells. Nat Rev Cancer. Jul; 2011 11(7):523-32. [PubMed: 21677677]

2. Creixell P, Schoof EM, Erler JT, Linding R. Navigating cancer network attractors for tumor-specific therapy. Nat Biotechnol. Sep; 2012 30(9):842-8. [PubMed: 22965061]

3. Barabasi AL, Gulbahce N, Loscalzo J. Network medicine: a network-based approach to human disease. Nat Rev Genet. Jan; 2011 12(1):56-68. [PubMed: 21164525]

4. Zhang B, Li H, Riggins RB, Zhan M, Xuan J, Zhang Z, Hoffman EP, Clarke R, Wang Y. Differential dependency network analysis to identify condition-specific topological changes in biological networks. Bioinformatics. Feb 15; 2009 25(4):526-32. [PubMed: 19112081]

5. Zhang B, Tian Y, Jin L, Li H, Shih IM, Madhavan S, Clarke R, Hoffman E, Xuan J, Hilakivi-Clarke L, Wang Y. DDN: A caBIG analytical tool for differential network analysis. Bioinformatics. 2011; 27(7):1036-1038. [PubMed: 21296752]

6. Ideker T, Krogan NJ. Differential network biology. Mol Syst Biol. 2012; 8

7. Hudson NJ, Dalrymple BP, Reverter A. Beyond differential expression: the quest for causal mutations and effector molecules. BMC Genomics. 2012; 13:356. [PubMed: 22849396] 
8. Hudson NJ, Reverter A, Dalrymple BP. A differential wiring analysis of expression data correctly identifies the gene containing the causal mutation. PLoS Comput Biol. May.2009 5(5):e1000382. [PubMed: 19412532]

9. de la Fuente A. From 'differential expression' to 'differential networking' - identification of dysfunctional regulatory networks in diseases. Trends Genet. Jul; 2010 26(7):326-33. [PubMed: 20570387]

10. Reverter A, Hudson NJ, Nagaraj SH, Perez-Enciso M, Dalrymple BP. Regulatory impact factors: unraveling the transcriptional regulation of complex traits from expression data. Bioinformatics. Apr 1; 2010 26(7):896-904. [PubMed: 20144946]

11. Lee MJ, Ye AS, Gardino AK, Heijink AM, Sorger PK, MacBeath G, Yaffe MB. Sequential application of anticancer drugs enhances cell death by rewiring apoptotic signaling networks. Cell. May 11; 2012 149(4):780-94. [PubMed: 22579283]

12. Skerker JM, Perchuk BS, Siryaporn A, Lubin EA, Ashenberg O, Goulian M, Laub MT. Rewiring the specificity of two-component signal transduction systems. Cell. Jun 13; 2008 133(6):1043-54. [PubMed: 18555780]

13. Weissleder R. Molecular imaging in cancer. Science. May 26; 2006 312(5777):1168-71. [PubMed: 16728630]

14. Peng H, Bateman A, Valencia A, Wren JD. Bioimage informatics: a new category in Bioinformatics. Bioinformatics. Apr 15.2012 28(8):1057. [PubMed: 22399678]

15. Chen L, Choyke PL, Chan TH, Chi CY, Wang G, Wang Y. Tissue-specific compartmental analysis for dynamic contrast-enhanced MR imaging of complex tumors. IEEE Trans Med Imaging. Dec; 2011 30(12):2044-58. [PubMed: 21708498]

16. Chen L, Chan TH, Choyke PL, Hillman EM, Chi CY, Bhujwalla ZM, Wang G, Wang SS, Szabo Z, Wang Y. CAM-CM: a signal deconvolution tool for in vivo dynamic contrast-enhanced imaging of complex tissues. Bioinformatics. Sep 15; 2011 27(18):2607-9. [PubMed: 21785131]

17. Yuan Y, Failmezger H, Rueda OM, Ali HR, Graf S, Chin SF, Schwarz RF, Curtis C, Dunning MJ, Bardwell H, Johnson N, Doyle S, Turashvili G, Provenzano E, Aparicio S, Caldas C, Markowetz F. Quantitative image analysis of cellular heterogeneity in breast tumors complements genomic profiling. Sci Transl Med. Oct 24.2012 4(157):157ra143.

18. Zhao B, Oxnard GR, Moskowitz CS, Kris MG, Pao W, Guo P, Rusch VM, Ladanyi M, Rizvi NA, Schwartz LH. A pilot study of volume measurement as a method of tumor response evaluation to aid biomarker development. Clin Cancer Res. Sep 15; 2010 16(18):4647-53. [PubMed: 20534736]

19. Albanese C, Rodriguez OC, VanMeter J, Fricke ST, Rood BR, Lee Y, Wang SS, Madhavan S, Gusev Y, Petricoin EF III, Wang Y. Preclinical Magnetic Resonance Imaging and Systems Biology in Cancer Research: Current Applications and Challenges. The American Journal of Pathology. 2013; 182(2):312-318. [PubMed: 23219428]

20. Sirajuddin P, Das S, Ringer L, Rodriguez OC, Sivakumar A, Lee YC, Üren A, Fricke ST, Rood B, Ozcan A, Wang SS, Karam S, Yenugonda V, Salinas P, Petricoin E, Pishvaian M, Lisanti MP, Wang Y, Schlegel R, Moasser B, Albanese C. Quantifying the CDK inhibitor VMY-1-103's activity and tissue levels in an in vivo tumor model by LC-MS/MS and by MRI. Cell Cycle. 2012; 11(20):3801-3809. [PubMed: 22983062]

21. Wang Y, Adali T, Xuan J, Szabo Z. Magnetic resonance image analysis by information theoretic criteria and stochastic site models. IEEE Trans Info Tech Biomed. 2001; 5(5):150-158.

22. Pierobon, M.; VanMeter, AJ.; Moroni, N.; Galdi, F.; Petricoin, EF. Molecular Profiling, Methods in Molecular Biology. Springer; 2012. Reverse-Phase Protein Microarrays; p. 215-235.

23. Beauchamp EM, Ringer L, Bulut G, Sajwan KP, Hall MD, Lee YC, Peaceman D, Ozdemirli M, Rodriguez O, Macdonald TJ, Albanese C, Toretsky JA, Uren A. Arsenic trioxide inhibits human cancer cell growth and tumor development in mice by blocking Hedgehog/GLI pathway. J Clin Invest. Jan 4; 2011 121(1):148-160. [PubMed: 21183792]

24. Segal E, Sirlin CB, Ooi C, Adler AS, Gollub J, Chen X, Chan BK, Matcuk GR, Barry CT, Chang HY, Kuo MD. Decoding global gene expression programs in liver cancer by noninvasive imaging. Nature Biotechnology. Jun; 2007 25(6):675-80.

25. Huang S, Li J, Chen K, Wu T, Ye J, Wu X, Yao L. A transfer learning approach for network modeling. IIE Transactions. Nov 01; 2012 44(11):915-931. [PubMed: 24526804] 
26. Tibshirani R. Regression shrinkage and selection via the lasso. Journal of the Royal Statistical Society, Series B. 1996; 58:267-288.

27. Zhang, B.; Wang, Y. Learning Structural Changes of Gaussian Graphical Models In Controlled Experiments. Conference on Uncertainty in Artificial Intelligence (UAI 2010); 2010.

28. Li H, Xuan J, Wang Y, Zhan M. Inferring regulatory networks. Front Biosci. 2008; 13:263-75. [PubMed: 17981545]

29. Tian, Y.; Zhang, B.; Shih, I-M.; Wang, Y. Knowledge-guided differential dependency network learning for detecting structural changes in biological networks. Proceedings of the 2nd ACM Conference on Bioinformatics, Computational Biology and Biomedicine; Chicago, Illinois. 2011. p. 254-263.

30. Bandyopadhyay S, Mehta M, Kuo D, Sung MK, Chuang R, Jaehnig EJ, Bodenmiller B, Licon K, Copeland W, Shales M, Fiedler D, Dutkowski J, Guenole A, van Attikum H, Shokat KM, Kolodner RD, Huh WK, Aebersold R, Keogh MC, Krogan NJ, Ideker T. Rewiring of genetic networks in response to DNA damage. Science. Dec 3; 2010 330(6009):1385-9. [PubMed: 21127252]

31. Kanehisa M, Goto S. KEGG: Kyoto Encyclopedia of Genes and Genomes. Nucleic Acids Research. Jan 1; 2000 28(1):27-30. [PubMed: 10592173]

32. I. CVX Research. CVX: Matlab software for disciplined convex programming, version 2.0 beta. Sep. 2012 http://cvxr.com/cvx

33. Grant, M.; Boyd, S. Graph implementations for nonsmooth convex programs, Recent Advances in Learning and Control (a tribute to M. Vidyasagar). In: Blondel, V.; Boyd, S.; Kimura, H., editors. Lecture Notes in Control and Information Sciences. Springer; 2008. p. 95-110.

34. Yuan X, Yu G, Hou X, Shih Ie M, Clarke R, Zhang J, Hoffman EP, Wang RR, Zhang Z, Wang Y. Genome-wide identification of significant aberrations in cancer genome. BMC Genomics. 2012; 13:342. [PubMed: 22839576]

35. Yu G, Zhang B, Bova GS, Xu J, Shih Ie M, Wang Y. BACOM: in silico detection of genomic deletion types and correction of normal cell contamination in copy number data. Bioinformatics. Jun 1; 2011 27(11):1473-80. [PubMed: 21498400]

36. Pinkel D, Albertson DG. Array comparative genomic hybridization and its applications in cancer. Nat Genet. Jun; 2005 37(Suppl):S11-7. [PubMed: 15920524]

37. Kuhn E, Wu RC, Guan B, Wu G, Zhang J, Wang Y, Song L, Yuan X, Wei L, Roden RB, Kuo KT, Nakayama K, Clarke B, Shaw P, Olvera N, Kurman RJ, Levine DA, Wang TL, Shih Ie M. Identification of molecular pathway aberrations in uterine serous carcinoma by genome-wide analyses. J Natl Cancer Inst. Oct 3; 2012 104(19):1503-13. [PubMed: 22923510]

38. Wang Y, Adali T, Kung SY, Szabo Z. Quantification and segmentation of brain tissues from MR images: a probabilistic neural network approach. IEEE Trans Image Processing. 1998; 7(8):11651181.

39. Van den Bulcke T, Van Leemput K, Naudts B, van Remortel P, Ma H, Verschoren A, De Moor B, Marchal K. SynTReN: a generator of synthetic gene expression data for design and analysis of structure learning algorithms. BMC Bioinformatics. 2006; 7(1):43. [PubMed: 16438721]

40. Loi S, Haibe-Kains B, Desmedt C, Lallemand F, Tutt AM, Gillet C, Ellis P, Harris A, Bergh J, Foekens JA, Klijn JGM, Larsimont D, Buyse M, Bontempi G, Delorenzi M, Piccart MJ, Sotiriou C. Definition of Clinically Distinct Molecular Subtypes in Estrogen Receptor-Positive Breast Carcinomas Through Genomic Grade. Journal of Clinical Oncology. Apr 1; 2007 25(10):1239_ 1246. [PubMed: 17401012]

41. Bergamaschi A, Katzenellenbogen BS. Tamoxifen downregulation of miR-451 increases 14-3-3 [zeta] and promotes breast cancer cell survival and endocrine resistance. Oncogene. 2012; 31(1):39-47. [PubMed: 21666713]

42. Bergamaschi A, Christensen B, Katzenellenbogen B. Reversal of endocrine resistance in breast cancer: interrelationships among 14-3-3zeta, FOXM1, and a gene signature associated with mitosis. Breast Cancer Research. 2011; 13(3):R70. [PubMed: 21707964]

43. Nowak MA, Komarova NL, Sengupta A, Jallepalli PV, Shih IM, Vogelstein B, Lengauer C. The role of chromosomal instability in tumor initiation. Proceedings of the National Academy of Sciences. Dec 10; 2002 99(25):16226-16231. 
44. Ricke RM, van Ree JH, van Deursen JM. Whole chromosome instability and cancer: a complex relationship. Trends in genetics: TIG. 2008; 24(9):457-466. [PubMed: 18675487]

45. Berman DM, Karhadkar SS, Hallahan AR, Pritchard JI, Eberhart CG, Watkins DN, Chen JK, Cooper MK, Taipale J, Olson JM, Beachy PA. Medulloblastoma growth inhibition by hedgehog pathway blockade. Science. Aug 30; 2002 297(5586):1559-61. [PubMed: 12202832]

46. de Bont JM, Packer RJ, Michiels EM, dBoer ML, Pieters R. Biological background of pediatric medulloblastoma and ependymoma: A review from a translational research perspective. NeuroOncology. Dec; 2008 10(6):1040-1060. [PubMed: 18676356]

47. Ideker T, Ozier O, Schwikowski B, Siegel AF. Discovering regulatory and signalling circuits in molecular interaction networks. Bioinformatics. 2002; 18(Suppl 1):S233-40. [PubMed: 12169552]

\section{Biographies}

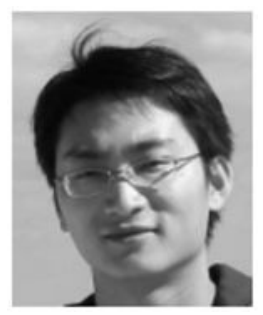

Ye Tian Is currently working towards the $\mathrm{PhD}$ degree under the supervision of Dr. Yue (Joseph) Wang, at the Department of Electrical and Computer Engineering, Virginia Tech. He received the BE and MS degree in Automation in 2007 and 2009 respectively, from Tsinghua University, Bejing, China.

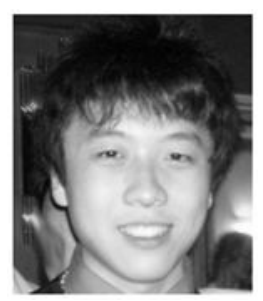

Sean S. Wang Is working towards BS degree at the Department of Electrical and Computer Engineering, University of Maryland College Park.

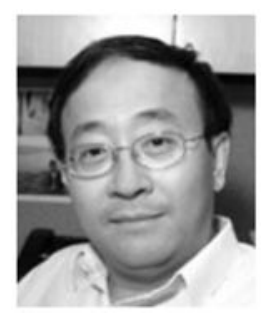

Zhen Zhang Is an Associate Professor of Pathology and Oncology at Johns Hopkins School of Medicine. 


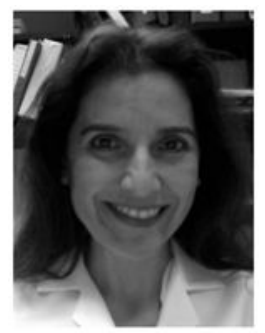

Olga C. Rodriguez Is an Assistant Professor in the Department of Oncology and CoDirector of the Preclinical Imaging Research Laboratory at the Lombardi Cancer Center at Georgetown University Medical Center. She received her M.D. degree from the Universidad del Rosario in Bogotá, Colombia and her Ph.D. in Biochemistry and Molecular Biology from Georgetown University. Her research interests include the advancement of preclinical imaging modalities for the characterization and evaluation of mouse models of disease, molecular mechanisms of prostate and brain cancer, and the advancement of Conditional Reprograming of Primary Cell Technology for the study of genitourinary malignancies.

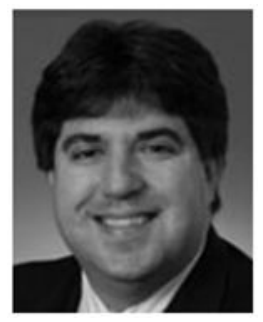

Emanuel Petricoin III Is Professor and Co-director of Center for Applied Proteomics and Molecular Medicine at George Mason University.

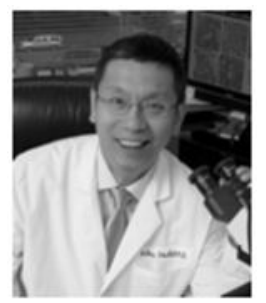

Ie-Ming Shih Is the Richard W. TeLinde Distinguished Professor of Pathology, Gynecology/Obstetrics and Oncology at the Johns Hopkins University School of Medicine. He is a board-certified attending pathologist in the Division of Gynecologic Pathology at the Johns Hopkins Hospital. Dr. Shih obtained his MD degree from the Taipei Medical University, Taiwan in 1988, and received his $\mathrm{PhD}$ in biomedical studies at the University of Pennsylvania in 1993. 


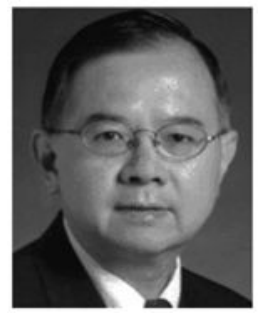

Daniel Chan Is Professor of Pathology, Oncology, Radiology, and Urology at the Johns Hopkins University School of Medicine, and Director of the Clinical Chemistry Division and Co-Director of the Pathology Core Laboratory of Johns Hopkins Hospital in Baltimore, Maryland. He is the also Director of the Biomarker Discovery Center, Johns Hopkins University. He received his BA degree in Biology from the University of Oregon and his $\mathrm{PhD}$ in Biochemistry from the State University of New York at Buffalo.

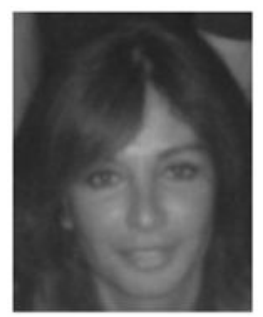

Maria Avantaggiati Is an Associate Professor at the Department of Molecular Oncology, Georgetown University. She received MD and PhD from University of Rome La Sapienza, Italy.

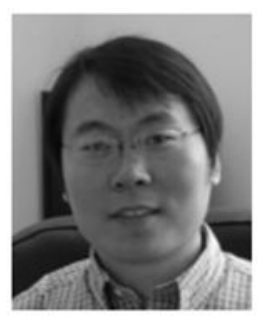

Guoqiang Yu Is an Assistant Professor at the Department of Electrical and Computer Engineering, Virginia Tech. He received PhD from Virginia Tech in 2011, MS from Tsinghua Univeristy, China in 2004 and BS from Shandong Univeristy, China in 2001.

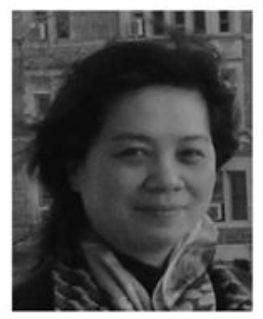


Shaozhen Ye Is a Professor at the college of Mathematics and Computer Science of Fuzhou University, China. She received PhD from Tsinghua University, China in 2004, MS and BS in 1995 and 1984 respectively, from Fuzhou University, China

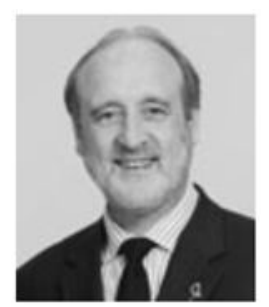

Robert Clarke Is Dean of research, Professor of Department of Oncology at Georgetown University. He received PhD and MSc from The Queen's University of Belfast, UK, BS from University of Ulster at Jordanstown, UK.

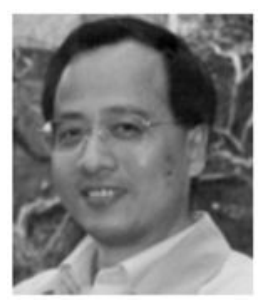

Chao Wang PhD, is with Beckman Institute for Advanced Science and Technology, University of Illinois at Urbana-Champaign.

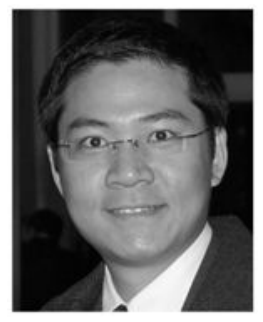

Bai Zhang Is a research associate at The Johns Hopkins University School of Medicine. He received PhD degree from Virginia Tech in 2011, MS and BS from Tsinhgua University, China in 2006 and 2004 respectively.

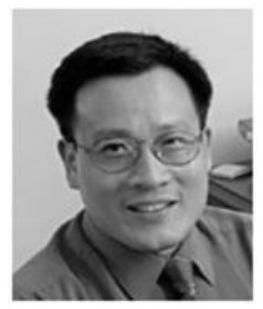


Yue Wang Is the Grant A. Dove Professor in the Department of Electrical and Computer Engineering, Virginia Tech. He received PhD from Universit of Maryland in 1995, MS and BS from Shanghai Jiaotong Univeristy, China in 1987 and 1984 respectively.

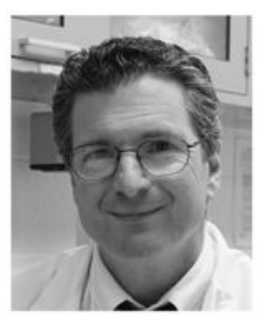

Chris Albanese Is Professor of Oncology and Pathology at Georgetown University Medical Center and is the Director of the Georgetown-Lombardi Preclinical Imaging Research Laboratory. He studies the roles of oncogenes, tumor suppressors and cell cycle regulatory proteins in the initiation and progression cancers and has an extensive background both in animal modeling and in molecular biology. He has have developed a comprehensive preclinical imaging program based on MRI and MR-Spectroscopy and is leading efforts to develop MR-Spectroscopy as a non-invasive method for assessing responses to chemotherapeutic drugs. In addition, he has been involved in the established a groundbreaking new technology referred to as conditionally reprogrammed cells (CRCs), which represent a revolutionary approach to continuous culture of both normal and malignant epithelial cells. He is building living biobank of matched tumor and normal adjacent epithelium from a variety of cancer sites, including GI, lung, pancreas, bladder and prostate as part of his programmatic initiative to use this system for mechanistic studies and perhaps more importantly, to personalized medicine. His goal is to enable the development of bedside to bench to bedside translational programs in cancer biology, imaging and precision therapy. 


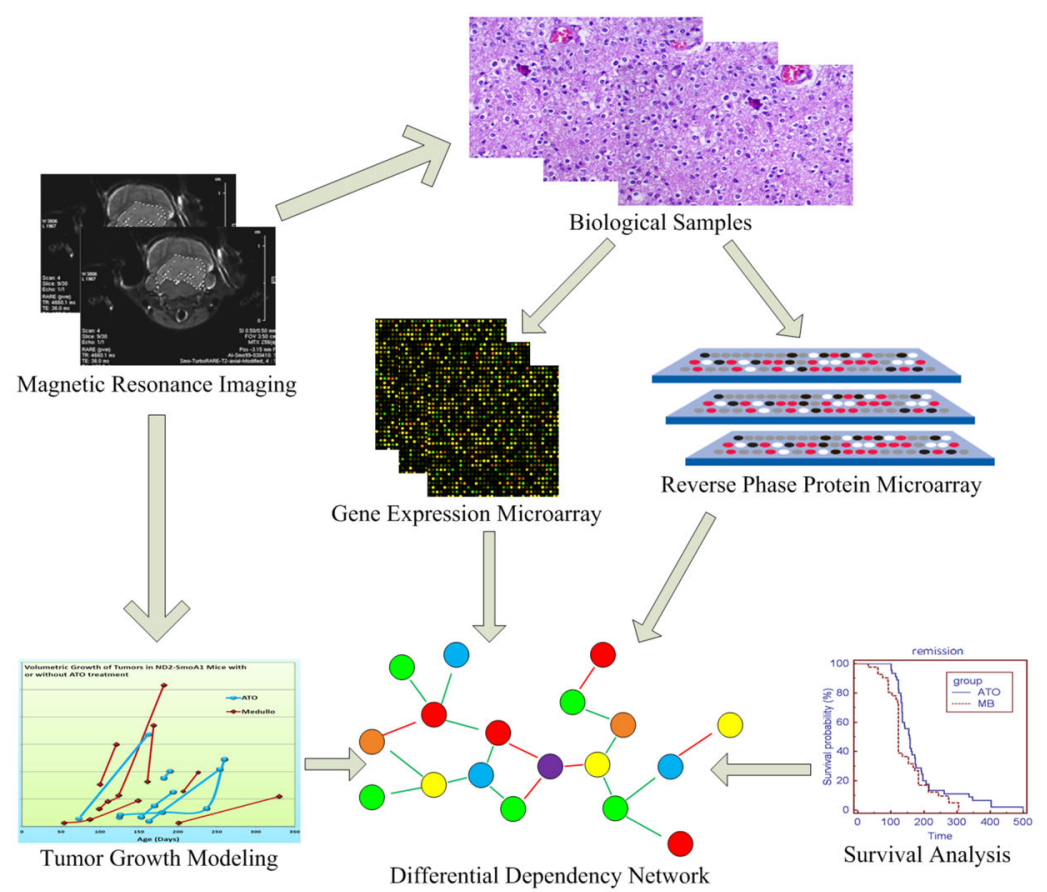

Fig. 1.

Overview of the data acquisition and analytics pipeline. 


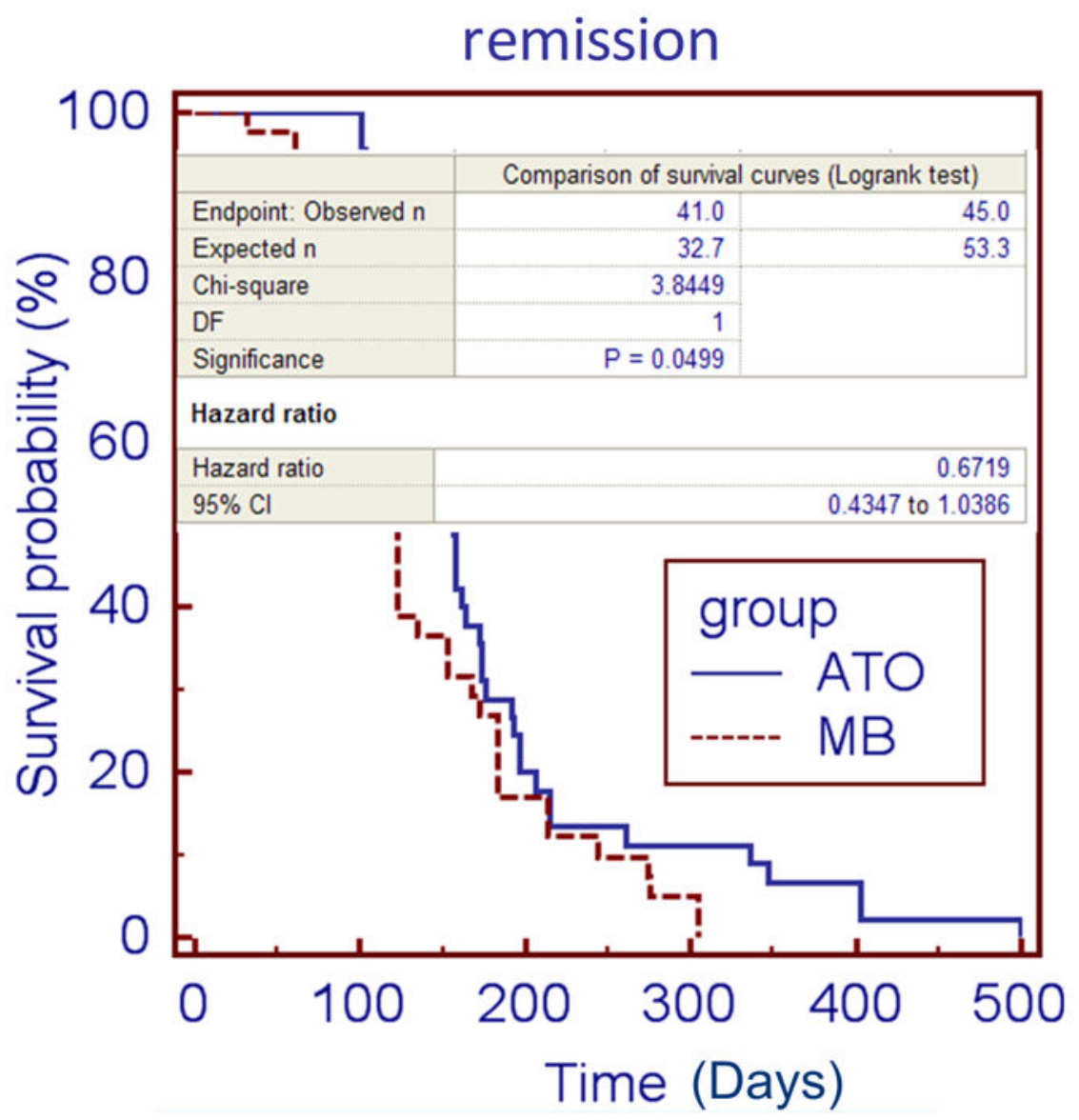

Fig. 2.

Survival analysis of MB mice model shows the significant improved survival of ATO treated samples. 


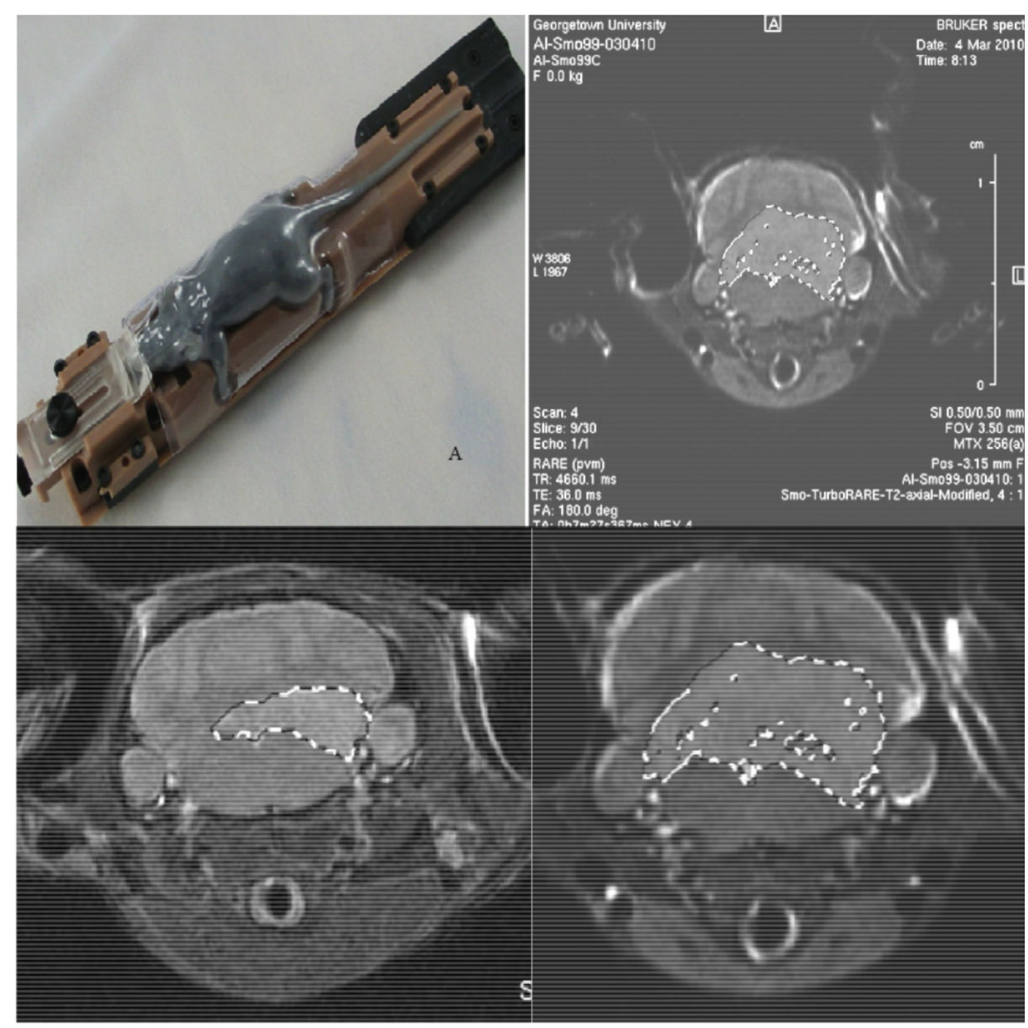

Fig. 3.

Longitudinal MRI of the ND2-SmoA1 MB mice. The upper-left corner shows the mouse experiment set. 


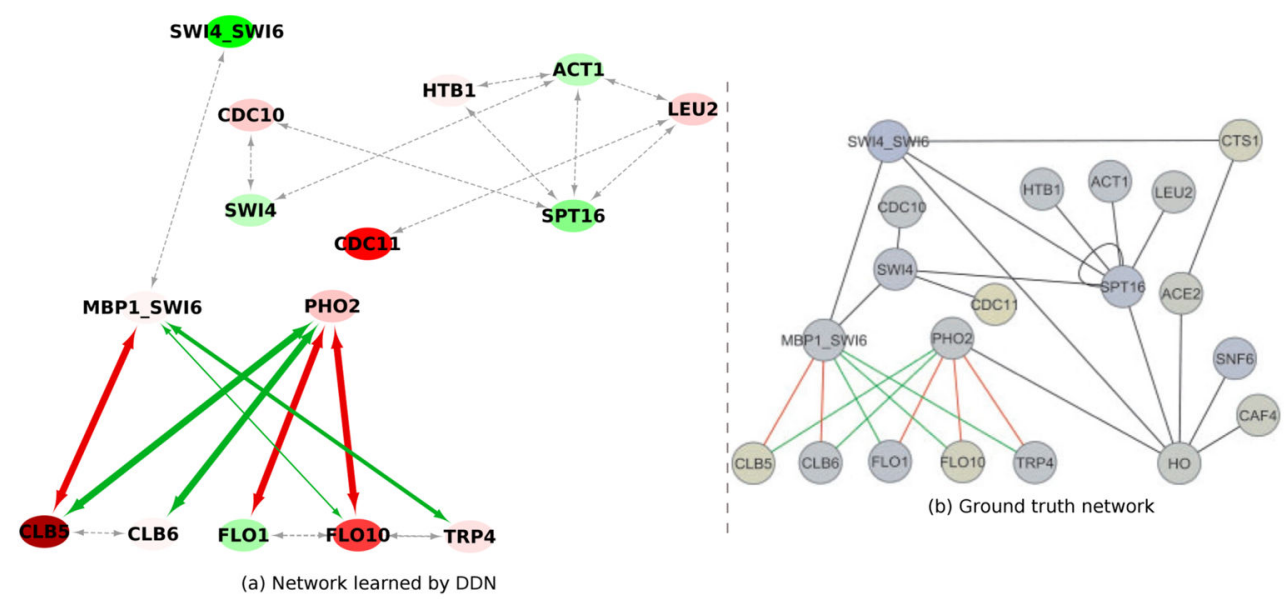

Fig. 4.

Simulation validation of DDN method of identifying differential and shared edges between two conditions. 


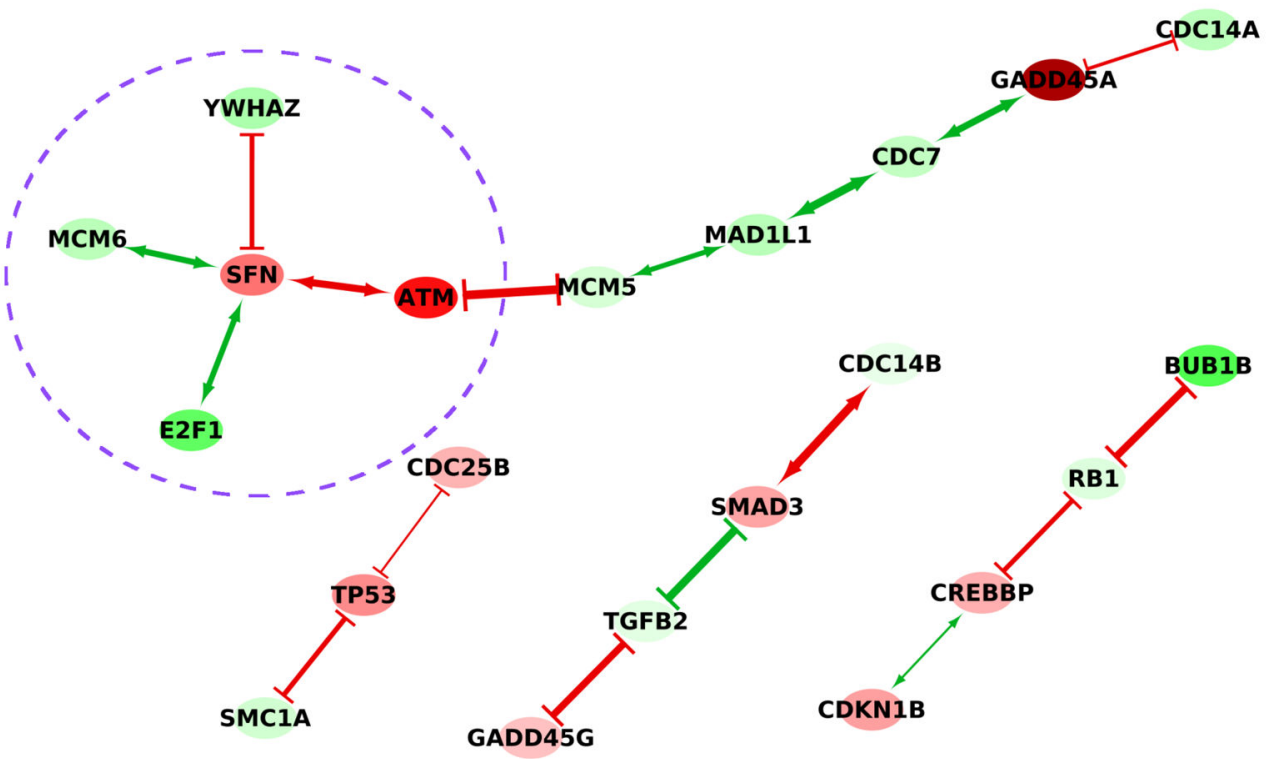

Fig. 5.

Differential network of breast cancer Cell Cycle pathway between early recurrent and nonrecurrent patients. 

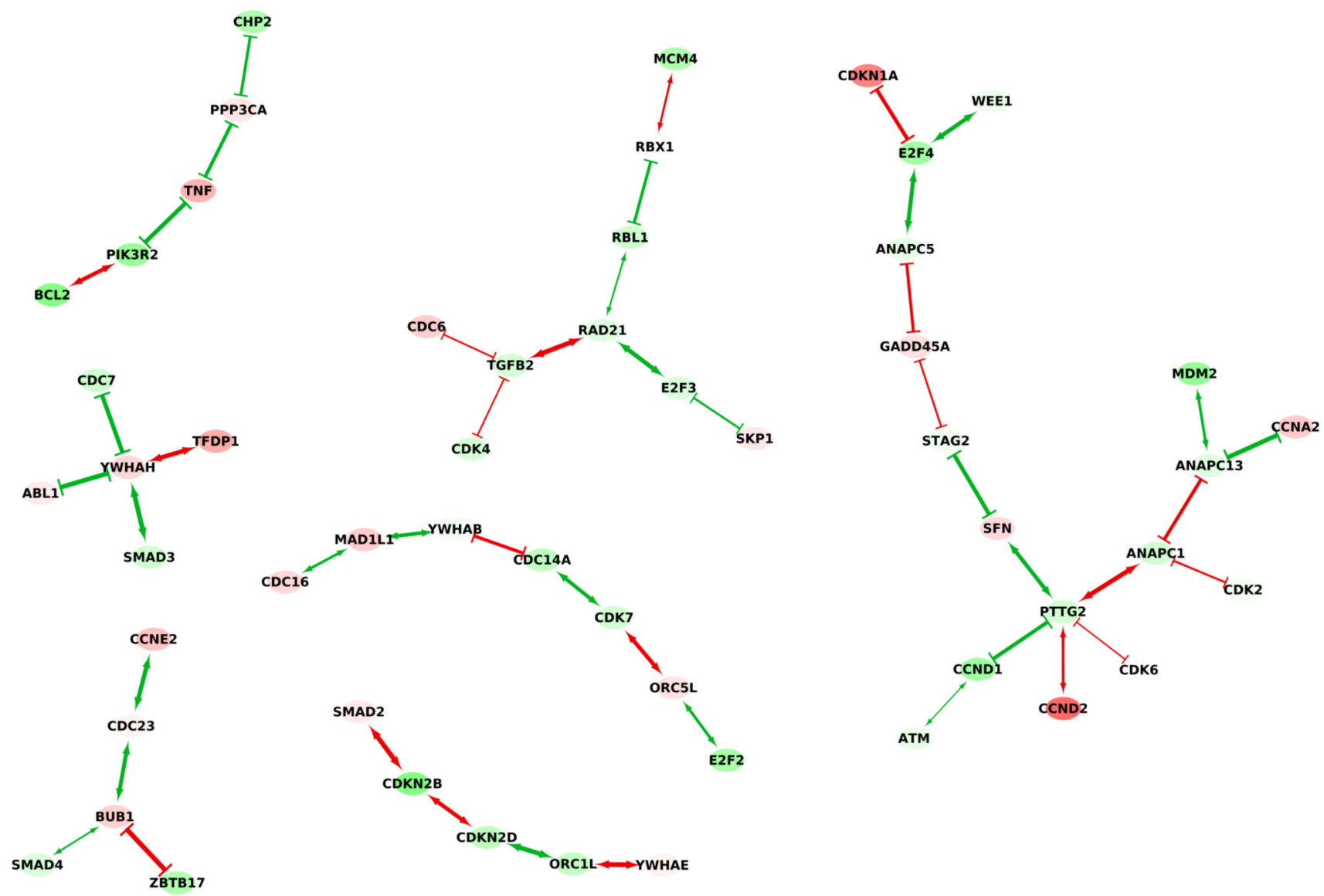

Fig. 6.

DDN result of chromosome stable and unstable groups on TCGA ovarian cancer data in Cell Cycle and Apoptosis pathways. Red edges are unstable groups. Green edges are stable groups. 


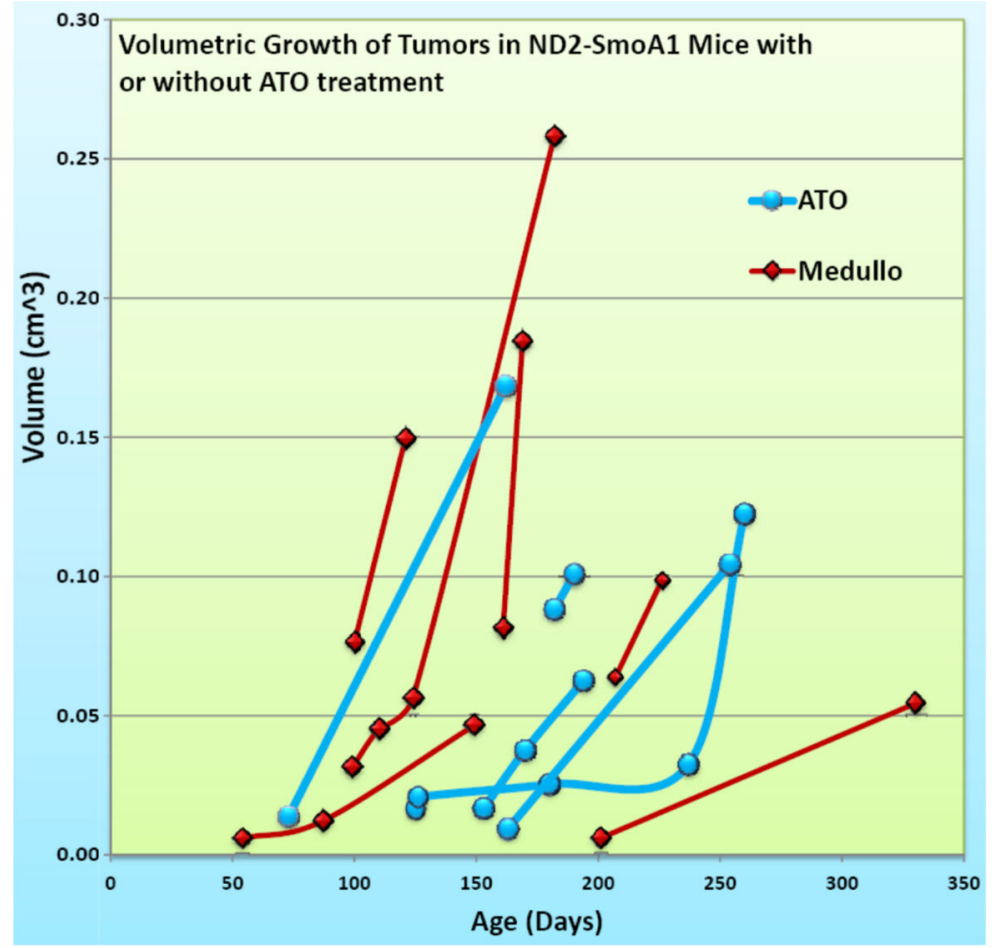

Fig. 7.

MRI-based tumor growth rates shows reduced growth rates in ATO treated samples. 

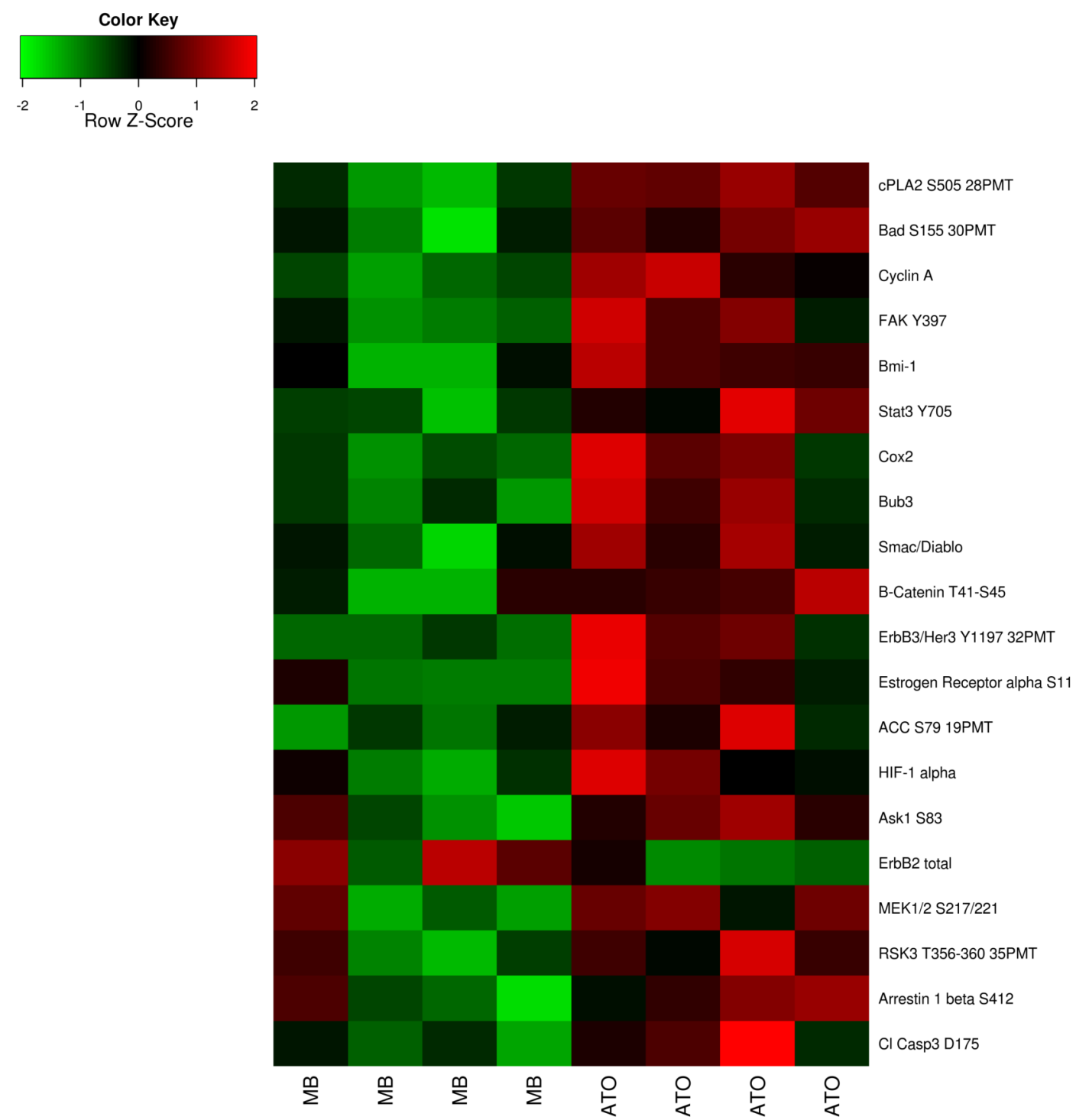

Fig. 8.

Differential analysis of RPMA data highlight the top 20 proteins modulated by ATO in vivo. 


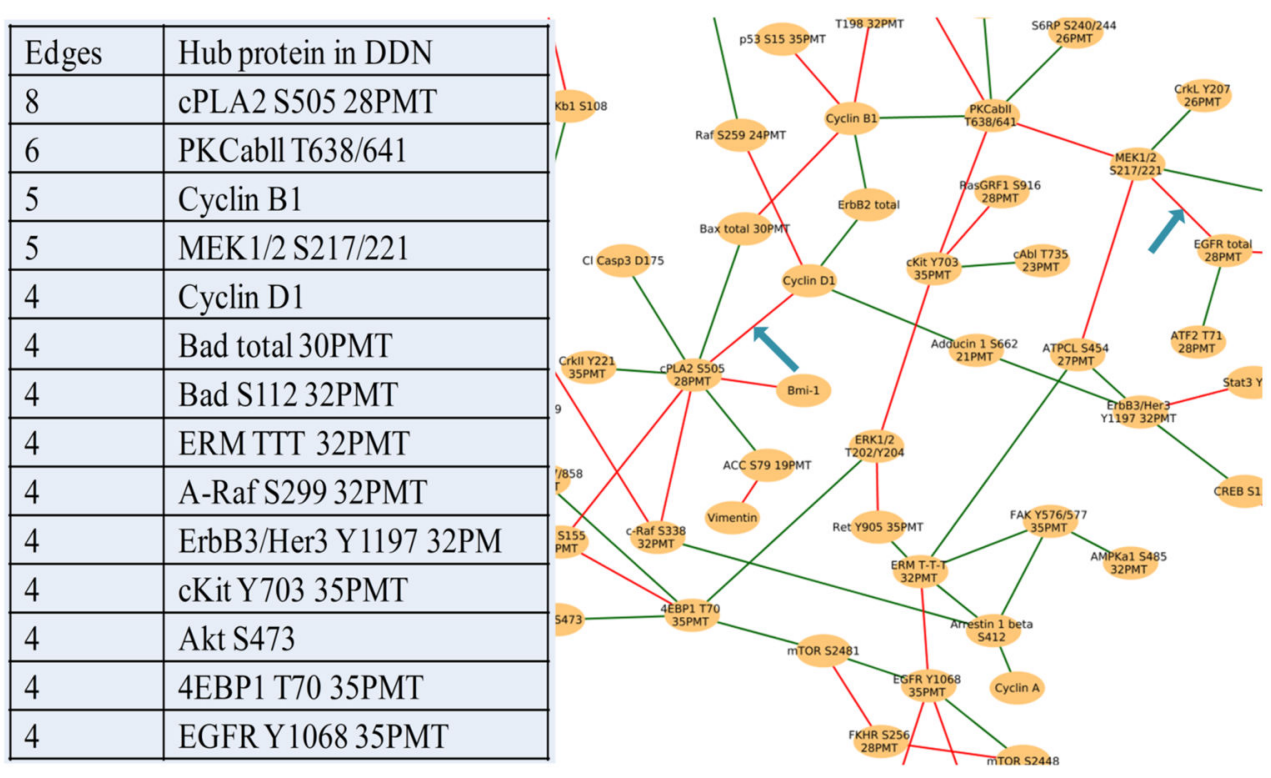

Fig. 9.

"Hubs" of the network rewiring by DDN analysis. Red edges are connections in MB destroyed by ATO treatment, and green edges are connections established after ATO treatment. 


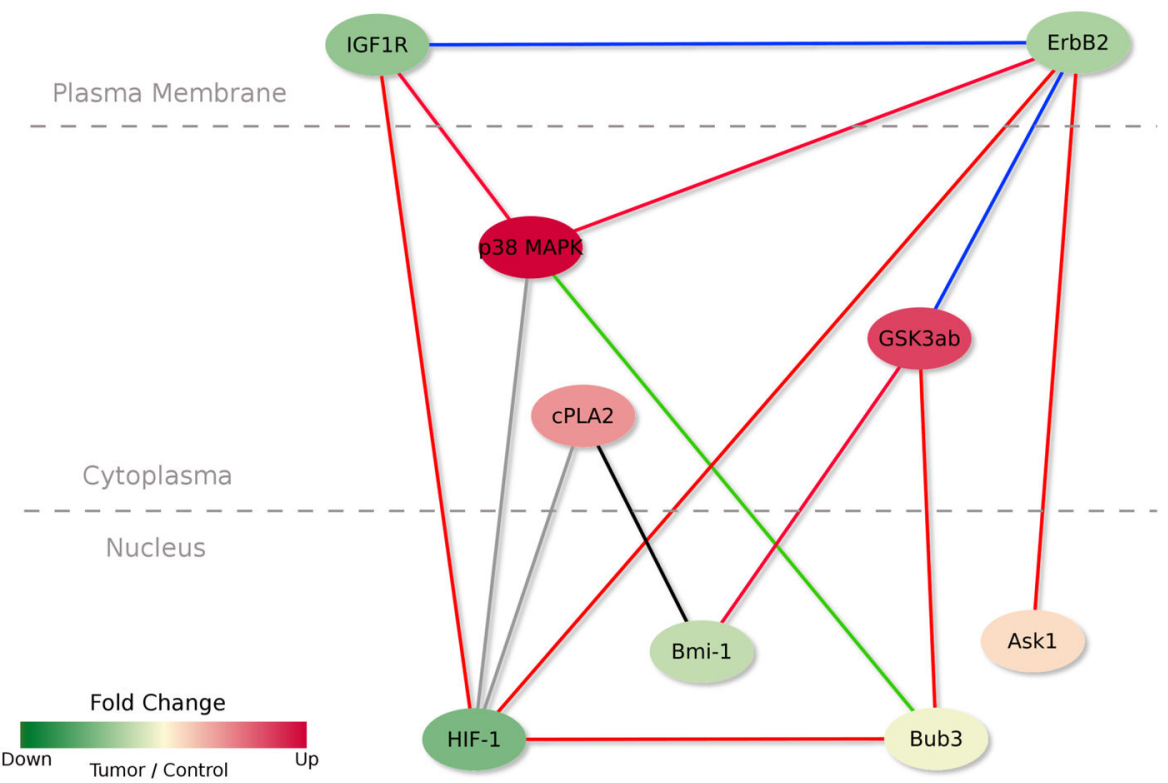

Fig. 10.

A focused network with proteins closely involved in MB signaling provides more insights into how ATO works to treat MB in vivo. Edges with different colors have specific meanings: blue - fully recovered by treatment (edges destroyed by tumor are regained); green - weakly created connection after treatment; red - not recovered; black - fully broken down tumor connection; gray - partially broken down tumor connection. 


\section{TABLE 1}

Tumor Growth Rate of Treated and Untreated Groups

\begin{tabular}{ccc}
\hline Slope (mm/day) & MB (6 samples) & ATO (5 samples) \\
\hline 0.003 & 0.005 \\
0.018 & 0.010 \\
0.129 & 0.011 \\
0.004 & 0.015 \\
0.028 & 0.017 \\
& 0.035 & \\
\hline Mean & $\mathbf{0 . 0 3 6}$ & $\mathbf{0 . 0 1 1 6}$ \\
\hline Standard deviation & $\mathbf{0 . 0 4 7}$ & $\mathbf{0 . 0 0 4 7}$ \\
\hline
\end{tabular}

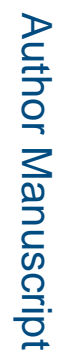

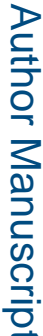

0.035 